\title{
Conflictos sociales en las áreas verdes urbanas en Lima Metropolitana
}

\author{
Jesús David Ramos Soto ${ }^{1}$ y José Manuel Ramos Soto² \\ Recibido: 4 mayo 2021 | Aceptado: 6 noviembre 2021
}

Los espacios verdes urbanos son de vital importancia para el desarrollo sostenible de las ciudades; pero al encontrarnos en un país con un constante crecimiento demográfico en las urbes, principalmente en Lima Metropolitana, se generan conflictos sociales debido a que la población busca nuevas zonas en donde habitar o porque surgen nuevos proyectos de infraestructura pública o privada que ponen en peligro la presencia de las áreas verdes en la ciudad.

Las áreas verdes urbanas contribuyen de manera positiva a la población y según López (2013), dichos espacios están muy vinculados en el bienestar social de la comunidad. Además, como lo plantea la Organización de las Naciones Unidas para la Alimentación y la Agricultura ([FAO], 2018), las áreas verdes en las zonas urbanas ofrecen beneficios necesarios para la sostenibilidad y mejorar la calidad de vida urbana, entre ellos podemos encontrar, la provisión y regulación de los flujos de agua, la mejora del microclima urbano, el almacenamiento y remoción de carbono, mejorar la calidad del aire e incrementar los servicios culturales.
Es importante mencionar que se deben respetar los espacios verdes establecidos en la ciudad y buscar que estos aumenten con la finalidad de alcanzar los Objetivos de Desarrollo Sostenible (Organización de las Naciones Unidas [ONU], 2021) que el Perú se comprometió a cumplir al año 2030, especialmente con el Objetivo 3 que se centra en la salud y bienestar; el Objetivo 11, enfocado a tener ciudades $y$ comunidades sostenibles; el Objetivo 13 cuyo fin es realizar acciones por el clima y el Objetivo 15 que vela por la vida de los ecosistemas terrestres.

Adicionalmente, es necesario reconocer que la Ordenanza Municipal $\quad \mathrm{N}^{\circ} 1852-M M L \quad$ (2014) establece el marco normativo para contribuir con la creación, conservación, protección, valoración, manejo, mantenimiento y sostenibilidad de las áreas verdes como elementos indispensables para mejorar la calidad de vida de las personas y del ambiente en la ciudad.

Según el Instituto Nacional de

\footnotetext{
${ }^{1}$ Universidad Nacional Agraria La Molina, Facultad de Ciencias Forestales; 20160182@lamolina.edu.pe ¿Universidad Nacional de Ingeniería, Facultad de Ingeniería Civil; jose.ramos.s@uni.pe
} 
Estadística e Información (INEI) el Perú, al 30 de junio del 2020, cuenta con 32625948 habitantes y Lima Metropolitana cuenta con aproximadamente $10 \quad$ millones habitantes, lo que conlleva a afirmar que cerca del tercio de la población peruana se encuentra en Lima Metropolitana. Cabe precisar que la población está expuesta a contaminantes del aire según el Informe Defensorial $\mathrm{N}^{\circ} 116$ elaborado en diciembre del 2006, sobre "La calidad del aire en Lima y su impacto en la salud y la vida de sus habitantes" ya que menciona que todos los estudios que se han realizado en Lima para cuantificar la calidad del aire, demuestran que la ciudad sufre un problema creciente de contaminación; es por ello que las áreas verdes son sumamente importantes para mejorar esta situación.

Respecto a la extensión de áreas verdes por habitante, en el informe Lima Como Vamos (2014), se indica que la extensión promedio en Lima Metropolitana es de 3,7 $\mathrm{m}^{2}$. Adicionalmente, según la ONU (2015), indica que se requiere una extensión mínima de $9 \mathrm{~m} 2 /$ habitante; en base a eso podemos apreciar que nos encontramos muy por debajo de los estándares internacionales respecto a áreas verdes por habitante.

A lo largo de la historia han surgido numerosos conflictos que han puesto en peligro a las áreas verdes urbanas, siendo uno de los más recientes, el que suscitó en el Bosque de Caja de Agua en el distrito de San Juan de Lurigancho (SJL). Dicho conflicto surgió en base a la propuesta realizada por el Ministerio de Vivienda, Construcción y Saneamiento (MVCS) para intervenir aproximadamente $15000 \mathrm{~m}^{2}$ de las $28639,77 \mathrm{~m}^{2}$ de este bosque urbano, que cumple la función de vivero forestal $y$ brinda diversos servicios ecosistémicos a la comunidad, para el establecimiento de 238 familias de la comunidad Shipibo-Conibo de Cantagallo.

Dicha acción causó una gran preocupación en la población, lo que conllevó a realizar protestas pacíficas en contra de la ejecución de dicho proyecto; ya que los vecinos reconocían la importancia ambiental e histórica que contribuye dicho espacio, que data de 1950, al distrito de SJL. Finalmente, el proyecto propuesto por el MVCS fue rechazado por los líderes de la comunidad Shipibo-Conibo; siendo un triunfo para la conservación de esta área verde. En base al movimiento vecinal y el de partes interesadas en defender esta área, actualmente, se ha iniciado el proceso para declarar este espacio como área de reserva ambiental y de llegar a concretarse, será de gran beneficio para la población.

Otro conflicto que ha surgido en torno a las áreas verdes ocurre en el distrito de Comas; donde se tiene contemplado realizar la ampliación del tramo norte del Metropolitano. Es importante mencionar que, según el informe Lima Como Vamos (2014), Comas cuenta con tan solo $3,9 \mathrm{~m}^{2}$ de área verde por habitante; evidenciando el déficit de áreas verdes en dicho distrito, lo que debe impulsar a realizar estrategias sólidas con el fin de incrementar dicho valor con el objetivo de mejorar la calidad del ambiente en 
beneficio de las personas.

Según la Municipalidad de Lima, indica que la ampliación incluirá la construcción de un corredor de 10,2 $\mathrm{km}$, desde la estación Naranjal del Metropolitano hasta la intersección de las avenidas Universitaria y Chimpu Ocllo en el distrito de Carabayllo. Esta ampliación, llegaría a intervenir aproximadamente 2,25 hectáreas del Parque Zonal Sinchi Roca, lo que conlleva afectar a alrededor de 5900 individuos arbóreos. Cabe mencionar que según el diario El Comercio (2019) dicho parque zonal ya ha sido anteriormente intervenido en el año 2009, dado que se realizaron construcciones para la implementación del patio de maniobras y cochera para los buses del Metropolitano; esta acción generó la pérdida de 7,8 hectáreas del parque zonal.

Respecto a la compensación ambiental, según la Municipalidad de Lima (2021) se tiene planificado plantar 6000 árboles de dos metros de altura en los distritos de Independencia, Comas y Carabayllo; pero esta compensación no puede ser comparada en la actualidad, dado que según la Sociedad Internacional de Arboricultura ([ISA], 2013) menciona que los beneficios medioambientales y económicos que produce un árbol joven son mínimos en comparación con los de un ejemplar adulto y es por ello que se debe buscar prolongar la vida de los árboles más grandes y adultos con un mantenimiento rutinario para multiplicar sus beneficios.

Dichos actos despertaron la indignación y preocupación de la población, puesto que ellos reconocen que dicho espacio trae consigo beneficios ambientales a la sociedad. Esto conllevó a que los vecinos se organicen creando la Asociación de Gestión y Defensa del Parque Zonal Sinchi Roca y Espacios Públicos, para realizar manifestaciones pacíficas buscando la protección de sus espacios verdes y ejecutar sesiones de capacitación para recibir información técnica de diversos profesionales.

En base a lo mencionado para estos dos casos, se sugiere realizar los procesos de planificación con participación ciudadana para que estos aporten de manera positiva a los proyectos a desarrollar; de esta manera, podrán recuperar la confianza en sus autoridades y sentir que realmente las obras públicas se realizan en favor de la población. Adicional a ello, se deberían realizar estudios de impacto ambiental (EIA) de manera responsable para poder identificar las consecuencias ambientales al alterar dichos ecosistemas y realizar las acciones correspondientes para poder incrementar la extensión de los espacios verdes en la urbe a fin de mejorar la calidad de vida de los ciudadanos.

En conclusión, podemos evidenciar que éstos son solo dos casos de muchos que ocurren en Lima Metropolitana y en todo el país; donde se pone en manifiesto la incompatibilidad de ciertos proyectos, que, si bien nacen en la búsqueda de nuevos espacios para la población, ponen en riesgo a las áreas verdes urbanas generando conflictos sociales. Asimismo, es importante asumir el rol de ser aliados estratégicos para evitar la reducción de nuestros pocos espacios verdes y hacer respetar la Ordenanza Municipal $N^{\circ} 1852-M M L$ (2014) que menciona que las áreas verdes son intangibles, inalienables e imprescriptibles. 


\section{LITERATURA CITADA}

Defensoría del Pueblo. (2006). Informe Defensorial N. ${ }^{\circ}$ 116: La Calidad del Aire en Lima y su Impacto en la Salud y la vida de sus Habitantes. www2.congreso.gob.pe/Sicr/ ApoyComisiones/

comision2011.nsf/021documentos/8B 420108E4101D0705258154005B4D7F/ \$FILE/Informe_N_116.pdf

Instituto Nacional de Estadística e Informática. (2020). Estado de la población peruana 2020. [Archivo PDF]. https://www.inei.gob.pe/ media/MenuRecursivo/ publicaciones_digitales/Est/Lib1743/ Libro.pdf

Jara, J. (12 de diciembre de 2019). Sinchi Roca: así fue mutilado el parque con el paso de los años. El Comercio. https://elcomercio.pe/ lima/obras/sinchi-roca-asi-fuemutilado-el-parque-con-el-paso-delos-anos-municipalidad-de-limametropolitano-comas-noticia/

Lima Como Vamos (2014). ¿Cómo Vamos en Ambiente?: Áreas Verdes. Asociación UNACEM. https:// www.limacomovamos.org/cm/wpcontent/uploads/2015/12/

InformeGestion2014.pdf

López, E. (2013). Beneficios en la implementación de áreas verdes urbanas para el desarrollo de ciudades turísticas. Revista de Arquitectura, Urbanismo y Ciencias Sociales, 4(1). https://xdoc. $\mathrm{mx} /$ documents/beneficios-en-la-

implementacion-de-areas-

verdes-5de2ce5e829e4
Ordenanza 1852 de 2014 [Municipalidad Metropolitana de Lima]. Ordenanza para la conservación y gestión de áreas verdes en la provincia de lima. 23 de diciembre de 2014. https:// apcvperu.gob.pe/files/Normatividad/ ORDENANZA_\% 20Nro_1852_MML_23_1_2014.pdf

Municipalidad Metropolitana de Lima. (s.f.). Ampliación Norte del Metropolitano. https:// aplicativos.munlima.gob.pe/ ampliacion-metropolitano

Organización de las Naciones Unidas (2015). Habitat III Issue Papers. https: / / uploads.habitat3.org/hb3/ Habitat-III-Issue-Paper-11_PublicSpace-2.0.compressed.pdf

Organización de las Naciones Unidas para la Alimentación y la Agricultura (2018). Bosques y ciudades sostenibles. Unasylva. Revista internacional sobre bosques $y$ actividades e industrias forestales, 69, 22-27. https://www.fao.org/3/ i8707es//8707ES.pdf

Organización de las Naciones Unidas (2021). Objetivos de desarrollo sostenible. https://www1.undp.org/ content/undp/es/home/sustainabledevelopment-goals.html

Sociedad Internacional de Arboricultura (2013). Beneficios de los árboles. [Archivo PDF]. https:// www.treesaregood.org/portals/0/ docs/treecare/Beneficios\%20de\%20los \%20Arboles.pdf 